University of Nebraska - Lincoln

DigitalCommons@University of Nebraska - Lincoln

$1-19-2008$

\title{
The bulk band structure and inner potential of layered $\ln _{4} \mathrm{Se}_{3}$
}

Jing Liu

University of Nebraska-Lincoln

Yaroslav B. Losovyj

University of Nebraska-Lincoln, ylozovyy@indiana.edu

Takashi Komesu

University of Nebraska-Lincoln, tkomesu2@unl.edu

Peter A. Dowben

University of Nebraska-Lincoln, pdowben@unl.edu

L. Makinistian

Universidad Nacional de Entre Ríos, 3101 Oro Verde (ER), Argentina

See next page for additional authors

Follow this and additional works at: https://digitalcommons.unl.edu/physicsdowben

Part of the Physics Commons

Liu, Jing; Losovyj, Yaroslav B.; Komesu, Takashi; Dowben, Peter A.; Makinistian, L.; Albinesi, E. A.; Petukhov, A. G.; Galiy, P.; and Fiyala, Ya., "The bulk band structure and inner potential of layered In4Se3" (2008). Peter Dowben Publications. 203.

https://digitalcommons.unl.edu/physicsdowben/203

This Article is brought to you for free and open access by the Research Papers in Physics and Astronomy at DigitalCommons@University of Nebraska - Lincoln. It has been accepted for inclusion in Peter Dowben Publications by an authorized administrator of DigitalCommons@University of Nebraska - Lincoln. 


\section{Authors}

Jing Liu, Yaroslav B. Losovyj, Takashi Komesu, Peter A. Dowben, L. Makinistian, E. A. Albinesi, A. G. Petukhov, P. Galiy, and Ya. Fiyala 
Published in Applied Surface Science (2008); doi:10.1016/j.apsusc.2008.01.061

Copyright (C) 2008 Elsevier B.V. Used by permission. http://www.sciencedirect.com/science/journal/01694332

Published online January 19, 2008.

\title{
The bulk band structure and inner potential of layered $\operatorname{In}_{4} \mathrm{Se}_{3}$
}

\author{
Jing Liu ${ }^{\text {a }}$, Ya. B. Losovyja, b, Takashi Komesu ${ }^{\text {a }}$, P. A. Dowben ${ }^{\text {a }}$, L. Makinistian ${ }^{\text {c, d }}$, \\ E. A. Albanesi c, d, A. G. Petukhove, P. Galiy ${ }^{f}$, and Ya. Fiyala ${ }^{f}$ \\ a Department of Physics and Astronomy and the Nebraska Center for Material and Nanoscience, \\ University of Nebraska-Lincoln, 116 Brace Laboratory, P.O. Box 880111, Lincoln, NE 68588, USA \\ b Louisiana State University, CAMD, 6980 Jefferson Highway, 70806 Baton Rouge, LA, USA \\ ${ }^{\text {c }}$ Facultad de Ingeniería, Universidad Nacional de Entre Ríos, 3101 Oro Verde (ER), Argentina \\ d INTEC-CONICET, Güemes 3450, 3000 Santa Fe, Argentina \\ e South Dakota School of Mines, Department of Physics, Rapid City, SD 57701-3995, USA \\ f Electronics Department, Lviv National University, 50 Dragomanov Street, 79005 Lviv, Ukraine \\ Corresponding author: P. Galiy - galiy@electronics.wups.lviv.ua ; tel. 380-322-964-678.
}

\begin{abstract}
The layered $\mathrm{In}_{4} \mathrm{Se}_{3}$ system does have a bulk band structure (i.e. discernable and significant band dispersion) perpendicular to the cleavage plane. Band widths (the extent of dispersion) of $300 \mathrm{meV}$ or more are observed, for In-p and Se-p weighted bands within the valence region, and is indicative of a bulk band structure. Two-dimensionality of state is clearly not conserved, and there must exist interactions between layers sufficient to support a bulk band structure.
\end{abstract}

Keywords: layered semiconductors, bulk band structure, photoemission, FP-LAPW

\section{Introduction}

Narrow band gap layered insulators have possible applications as the thin gate dielectric in tunnel junctions and other related structures. Recently, non-oxide layered materials like hexagonal boron nitride have been considered [1]. At issue is whether such materials are truly two-dimensional, as is generally implied [2, 3, 4] or exhibit a bulk band structure perpendicular to the cleavage plane. The studies of $\mathrm{In}_{4} \mathrm{Se}_{3}$ semiconductor crystal cleavage surfaces and interface formation are interesting from this point of view as this material crystallizes in a layered structure with, in principle, weak interaction of van der Waals type between the layers and strong covalent-ionic interactions within the layer. The quasi two-dimensionality (2D) of layered crystals and, consequently, absence of free unsaturated electron bonds on the cleavage surfaces, does not quite apply as the layers are not perfectly flat but warped [5-8]. $\mathrm{In}_{4} \mathrm{Se}_{3}$ as in fact is dominated by an $\left(\operatorname{In}_{3}\right)^{5^{+}}$multivalent clusters bonded with selenium through ionic covalent bonds (see Figure 1). $\mathrm{In}_{4} \mathrm{Se}_{3}$ seems to be less studied among the layered chalcogenides crystals $[2,3]$, yet continues to attract attention as a natural low dimensional $2 \mathrm{D}$ material in much the same way as occurs with layered systems with flat layers, held together with van der Waals forces.

Here we show that this $\operatorname{In}_{4} \mathrm{Se}_{3}$ system does have a bulk band structure (i.e. discernable and significant band dispersion) perpendicular to the cleavage plane and thus is not two-dimensional.

\section{Experimental details}

The layered crystal structure of $\operatorname{In}_{4} \mathrm{Se}_{3}$ allows one to a obtain cleaved ( 10 o $)$ ) surface (Figure 1). The crystals were grown by Czochralski method, and then cleaved in situ. Surface order was established by low energy electron diffraction.

Angle-resolved photoemission spectra were obtained using plane polarized synchrotron light dispersed by a $3 \mathrm{~m}$ toroidal grating monochromator $[9,10]$, at the Center for Microstructures and Devices (CAMD) [11]. The measurements were made in a UHV chamber employing a hemispherical electron analyzer with an angular acceptance of $\pm 1^{\circ}$, as described elsewhere $[9,10]$. The combined resolution of the electron energy analyzer and monochromator is $120-150$ 


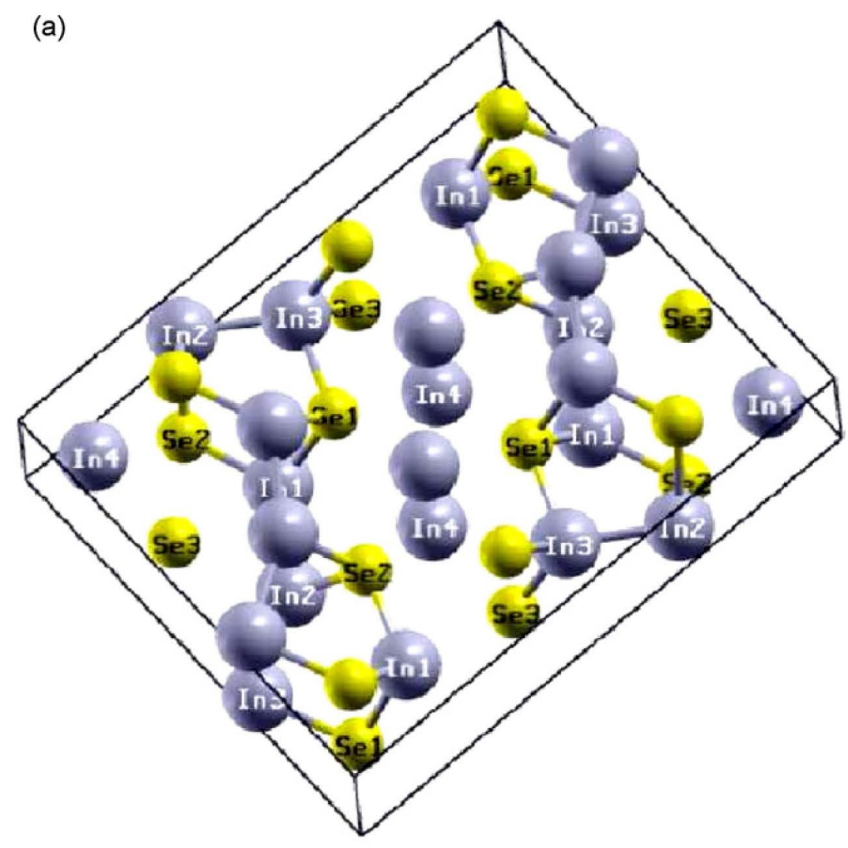

(b)

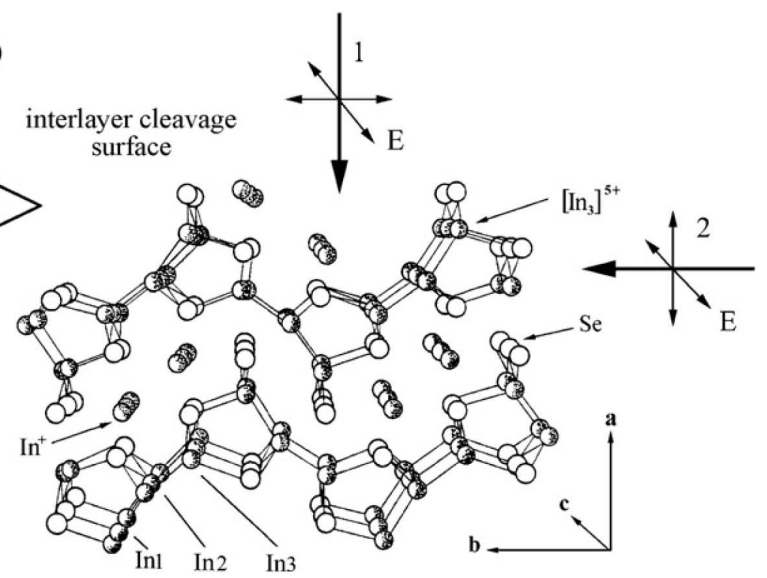

Figure 1. The schematic crystal structure for $\operatorname{In}_{4} \mathrm{Se}_{3}$ is presented top. The lattice constants of the crystal structure are: $a=15.296(1) \AA$; $b=12.308(1) \AA$; $c=4.0806(5) \AA$; space group $P_{\mathrm{nnm}}$ [5-8], dominated by an $\left(\operatorname{In}_{3}\right)^{5^{+}}$multivalent clusters bonded with selenium through ionic covalent bonds. The cleavage direction is indicated at the bottom, by the triangle in the upper left.

meV for higher photon energies $(50-120 \mathrm{eV})$, but higher resolution (about $80 \mathrm{meV}$ ) is obtained at lower photon energies of $15-40 \mathrm{eV}$. The photoemission experiments were undertaken with a light incidence angle of $45^{\circ}$ with respect to the surface normal and with the photoelectrons collected along the surface normal, to preserve the highest point group symmetry and eliminate any wave vector component parallel with the surface. All binding energies are referenced to the Fermi level, as determined from clean gold. The bulk band mapping was undertaken at room temperature, well above the Curie temperature.

\section{Theory and structure}

The $\mathrm{In}_{4} \mathrm{Se}_{3}$ crystal structure according to [5-8] (projection of ( $\left.\begin{array}{lll}0 & 0 & 1\end{array}\right)$ plane) is shown in Figure 1. The measured lat- tice constants of the crystal structure are: $a=15.297(1) \AA$; $b=12.308(1) \AA ; c=4.0810(5) \AA$ in the orthorhombic space group $P_{\mathrm{nnm}}$, obtained by X-ray diffraction using $\mathrm{Cu} \mathrm{Ka}$ radiation. These values are in agreement with the published values of $a=15.296(1) \AA ; b=12.308(1) \AA ; c=4.0806(5) \AA$; also in the space group $P_{\mathrm{nnm}}$ [8]. The interlayer cleavage plane $(100)$ of $\operatorname{In}_{4} \mathrm{Se}_{3}$ is normal to the axis of crystal growth $a$. The triangle in the upper left shows the cleavage direction (Figure $1 \mathrm{~b}$ ).

We have performed FP-LAPW calculations of $\operatorname{In}_{4} \mathrm{Se}_{3}$ within the density functional theory (DFT), in the form implemented in the WIEN2k code [12]. As part of our treatment, we have used the so-called generalized gradient approximation (GGA) [13, 14], in the formal parameterization scheme of Perdew-Burke-Ernzerhof (PBE) [15]. Our calculations were performed on the basis of structural information taken from Schwarz et al. [8]. We have used 120 irreducible $k$-points in the Brillouin zone for the self-consistent calculation, with an $R_{\mathrm{MT}} K_{\text {max }}$ product of 7 , here $R_{\mathrm{MT}}$ is the smallest of all atomic sphere radii and $K_{\max }$ is the planewave cut-off. The system has 28 (i.e. $7 \times 4$ ) atoms in the unit cell with seven crystallographically inequivalent sites (four indium and three selenium), each of which occupying four equivalent positions (see Figure 1a). In general, our results are comparable with prior calculations [16].

The electronic structure of $\operatorname{In}_{4} \mathrm{Se}_{3}$ can be understood as five main bundles of bands (four below and one above the Fermi energy). There are flat isotropic occupied bands due to the In-4d orbitals between -15.00 and $-13.73 \mathrm{eV}$ binding energies, followed by a less "populated" band due to Se-4s and also (though less significant) In-4d orbitals between -13.59 and $-11.91 \mathrm{eV}$ binding energy. Then there is an empty (unoccupied) region between -11.91 and $-7.17 \mathrm{eV}$ below the Fermi level, where the bottom of the valence band begins. The valence band maximum is dominated by In-5s, In-5p, Se- $4 \mathrm{p}$, while at $-1.5 \mathrm{eV}$ binding energy the band is more dominated by In- $5 \mathrm{p}$. The bands at -2.5 to $-4.5 \mathrm{eV}$ binding energy are dominated by the In- $5 \mathrm{p}$ and Se- $4 \mathrm{p}$ orbitals. The calculated density of occupied states, within the valence band region, is seen to be in good agreement with the photoemission, as indicated in Figure 2.

The conduction band is mainly p-like (with both In and Se contributions). However there is a sharp peak of In-s orbitals near the bottom of the conduction band. A similar situation holds for the narrow In-s states near the valence band maximum. These s-cation states, hybridized with their p-cation and anion counterparts, are very important for the physical properties of this material, since they define the band gap.

\section{The bulk band structure}

Figure 3 illustrates the dependence of the normal emission photoemission spectra on photon energy from 15 to $40 \mathrm{eV}$. From the dispersion of the bands, evident in the photoemission spectra as a function of photon energy, we have been able to construct a band structure mapping along the $\Gamma Z$ line (along the surface normal). The perpendicular component of the crystal wave vector $\left(k_{\perp}\right)$, however, is not conserved across the solid vacuum interface because of the 

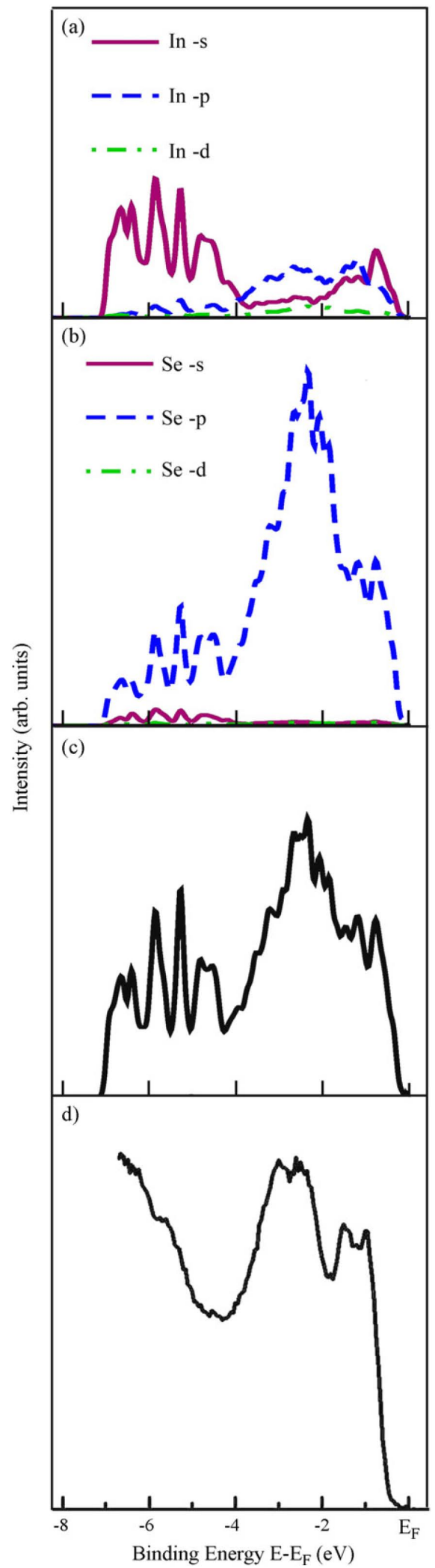

Figure 2. The partial In (a) and Se (b) occupied density of states provide a total density of states (c) that are compared to the photoemission (d). The photoemission spectra were taken with a combined instrumental resolution of $70 \mathrm{meV}$, at a photon energy of $15 \mathrm{eV}$, with a light incidence angle of $45^{\circ}$ with respect to the surface normal and with the photoelectrons collected along the surface normal.

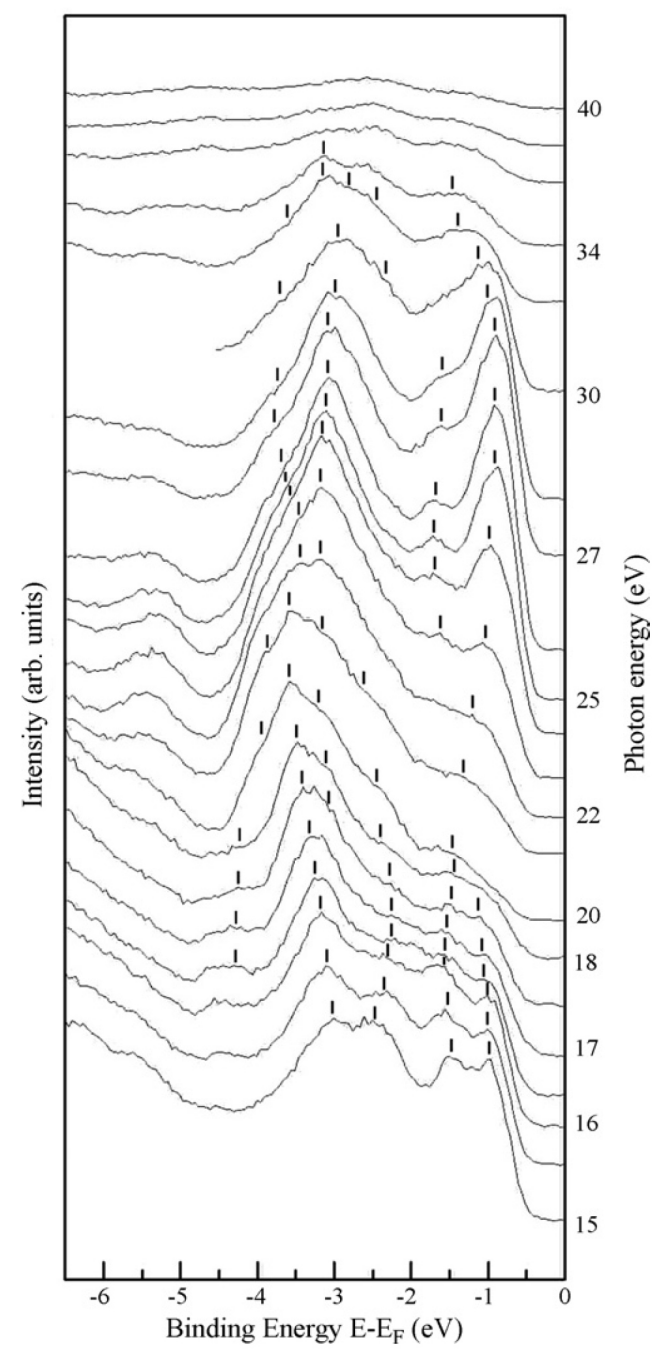

Figure 3. The photoemission spectra of $\operatorname{In}_{4} \mathrm{Se}_{3}(\mathrm{O}$ o 1$)$ for photon energies from 14 to $40 \mathrm{eV}$, taken at normal emission $\left(k_{\perp}=0\right)$.

crystal truncation at the surface. Thus the perpendicular wave vector in the crystal can be determined using

$$
k_{\perp}=\left[\left(\frac{-2 m}{\hbar^{2}}\right) E_{\mathrm{kin}}[\cos (\theta)]^{2}+U_{\mathrm{in}}\right]^{1 / 2}
$$

where $\theta$ is the emission angle of the photoelectron and $U_{\text {in }}$ is the inner potential of the solid $[17,18]$.

Following Equation (1), the resulting experimental band structure has been plotted in Figure 4 along the $\left\langle\begin{array}{lll}0 & 0 & 1\end{array}\right\rangle$ direction, or from $\Gamma$ to $\mathrm{Z}$ of the bulk Brillouin zone (along the surface normal). At photon energies of 14 and $27 \mathrm{eV}$, where the band at the valence band maximum has the least binding energy, should be the photon energies where the wave vectors correspond to the $\Gamma$ point in the $k$ vector space. This corresponds to a $\Gamma$ point occurring at $2.35 \AA^{-1}$, while the zone edge occurs at 1.91 and $2.76 \AA^{-1}$. This suggests a lattice constant of $7.4 \AA$, or about $1 / 2$ the expected lattice constant of $15.3 \AA$. This is different from the in plane band dispersion, where the critical points are consistent with both expectation and the low energy electron diffraction as will be discussed in detail elsewhere [19]. 


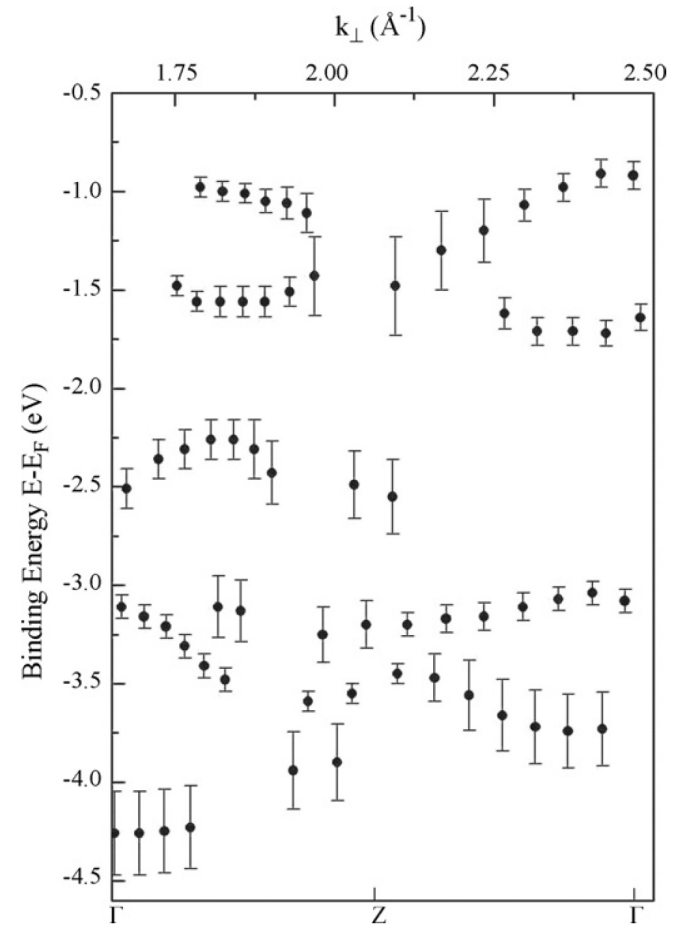

Figure 4. Experimental bulk band structure of $\operatorname{In}_{4} \mathrm{Se}_{3}$ along the $\langle\mathrm{o} O$ 1) direction. From the experimental data, both critical points and the experimental wave vectors are identified.

Though the $\mathrm{Z}$ point in Figure 4 should designate another $\Gamma$ point in the bulk band structure, surprisingly, it does not appear to do so. We may understand this smaller than expected lattice constant in the bulk band structure if both "a" and "b" cleavage surfaces are present at the surface (separated by $1 / 2$ a unit cell along the surface normal or about $7.65 \AA$ ). By superposition, the bulk band structure may now resemble the bulk band structure of a crystal with a glide plane symmetry, as in $\mathrm{CoS}_{2}$ [20]. This increase in the effective Brillouin zone, due to superposition of different facets or cleavage surfaces, along the surface normal would tend to diminish the extent of the band dispersion measured, making the observation of bulk band structure even more significant. If we now assume an effective lattice constant along the surface normal of $15.3 \AA / 2$ (although not the correct lattice constant), we can estimate that the inner potential $U$ of about $2.5 \mathrm{eV}$ if the critical point at $2.35 \AA^{-1}$ is the "effective" $\Gamma_{3}$ and about $21 \mathrm{eV}$ if this is the effective $\Gamma_{4}$ critical point. Neither value would be expected for a conventional narrow band gap insulator.

\section{Summary}

The downward dispersion (towards greater binding energy) of the band at the valence band maximum, away from the $\Gamma$ point, can be understood as this band is dominated by In-s and $\mathrm{Se}-\mathrm{p}_{y}$ while band at $-1.5 \mathrm{eV}$ binding energy has strong $\mathrm{p}_{y} \mathrm{p}_{z}$ components. In any case the band widths (the extent of dispersion) of $300 \mathrm{meV}$ or more, for bands within the valence region, is well more than the instrumental resolution and is indicative of a bulk band structure. Two-dimensionality of state is clearly not conserved; there must there- fore exist interactions between layers sufficient to support a bulk band structure.

\section{Acknowledgements}

This research was supported through the ONR Grant No. Noo014-06-1-0616, the Nebraska Research Initiative and the NSF "QSPINS" MRSEC (DMR 0213808) at UNL. The Center for Advanced Microstructures and Devices is supported by the Louisiana Board of Regents. The support from CONICET, UNER, and ANPCyT, Argentina is also acknowledged with appreciation.

\section{References}

[1] R. A. Lukaszew, Y. Sheng, C. Uher, and R. Clarke, Appl. Phys. Lett. 75 (1999), p. 1941.

[2] W. Jaegermann, A. Klein, and C. Pettenkofer In: H.P. Hughes and H. I. Starnberg, Editors, Physics and Chemistry of Materials with Low-Dimensional Structures, vol. 24, Kluwer Academic Publishers, Dordrecht (2000), pp. 317-402.

[3] O. A. Balitskii, V. P. Savchyn, B. Jaeckel, and W. Jaegermann, Phys. E: Low Dimensional Syst. Nanostruct. 22 (2004), p. 921.

[4] A. Ates, B. Gurbulak, M. Yildirim, and S. Dogan, Phys. E: Low Dimensional Syst. Nanostruct. 16 (2003), p. 274.

[5] J. H. C. Hogg, H. H. Sutherland, and D. J. Wiloans, Acta Crystallogr. B 29 (1973), p. 1590.

[6] A. Likfomann and J. Eitienne, C.R. Acad. Sci. C 275 (1972), p. 1907.

[7] L. I. Man, R. M. Imamov, and S. A. Semiletov, Crystalographij 21 (1976), p. 628.

[8] U. Schwarz, H. Hillebrecht, H. J. Deiseroth, and R. Walther, Zeitschrift für Kristallographie 210 (1995), p. 342.

[9] Ya. Losovyj, I. Ketsman, E. Morikawa, Z. Wang, J. Tang, and P. Dowben, Nucl. Instrumen. Methods Phys. Res. A 582 (2007), pp. 264-266.

[10] P. A. Dowben, D. LaGraffe, and M. Onellion, J. Phys.: Condens. Matter 1 (1989), p. 6571.

[11] J. Hormes, J. D. Scott and V. P. Suller, Synchrot. Radiat. News 19 (2006), p. 27.

[12] P. Blaha, K. Schwarz, J. Luitz, Vienna University of Technology, 2001. (Improved and updated version of the WIEN code, published by P. Blaha, K. Schwarz, P. Sorantin, and S. B. Rickey, Comp. Phys. Commun. 59 (1990) 399.).

[13] J. P. Perdew, J. A. Chevary, S. H. Vosko, K. A. Jackson, M. R. Pederson, D. J. Sing, and C. Fiolhais, Phys. Rev. B 46 (1992), p. 6671.

[14] J. P. Perdew and Y. Wang, Phys. Rev. B 45 (1992), p. 13244.

[15] (a)J. P. Perdew, K. Burke, and M. Ernzerhof, Phys. Rev. Lett. 77 (1996), p. 3865.

(b)J. P. Perdew, K. Burke and M. Ernzerhof, Phys. Rev. Lett. 78 (1997), p. 1396.

[16] M. Szanjder, K. Z. Rushchanskii, L. Yu. Kharkhalis, and D. M. Bercha, Phys. Status Solidi B $\mathbf{2 4 3}$ (2006), p. 592.

[17] E. W. Plummer and W. Eberhardt, Adv. Chem. Phys. 49 (1982), p. 533 .

[18] L. J. Clarke, Surface Crystallography: An Introduction to Low Energy Electron Diffraction, Wiley, New York (1985).

[19] Ya. B. Losovyj, J. Liu, T. Komesu, P. Galiy, A. Ciszewski, P. Mazur, S. Zuber, L. Makinistian, E. A. Albanesi, A. Petukhov, and P. A. Dowben, Surface structure and band mapping of the $\operatorname{In}_{4} \mathrm{Se}_{3}$ semiconductor surface, (in preparation).

[20] N. Wu, Ya. B. Losovyj, D. Wisbey, K. Belashchenko, M. Manno, L. Wang, C. Leighton, and P. A. Dowben, J. Phys.: Condens. Matter 19 (2007), p. 156224. 